\title{
Food or Food Product
}

National Cancer Institute

\section{Source}

National Cancer Institute. Food or Food Product. NCI Thesaurus. Code C1949.

Processed or unprocessed substances obtained from animal, plant, microorg anism and mining source that provide nutrients for living org anisms to maintain biological processes or functions. 